\title{
Bioinspired Repellent Pipette Tips with Low Retention Properties Prevent Contamination in Handling Biological Samples
}

Hanie Yousefi

University of Toronto

Amid Shakeri ( $\square$ shakeria@mcmaster.ca )

McMaster University

Samer Kullab

McMaster University

Dalya Al-Mfarej

McMaster University

Martin Rottman

Versailles Saint-Quentin-en-Yvelines University

\section{Tohid Didar}

McMaster University

\section{Research Article}

Keywords: Lubricant-infused pipette tips, Fluorosilanization, Omniphobic properties, Carryover residue

Posted Date: December 9th, 2021

DOI: https://doi.org/10.21203/rs.3.rs-1129108/v1

License: (9) (i) This work is licensed under a Creative Commons Attribution 4.0 International License. Read Full License 


\section{Abstract}

Cross-contamination of biological samples during handling and preparation, is a major issue in laboratory setups, leading to false-positives or false-negatives. Sample carryover residue in pipette tips contributes greatly to this issue. Most pipette tips on the market are manufactured with hydrophobic polymers that are able to repel high surface tension liquids, yet they lack in performance when low surface tension liquids are involved. This presents an obstacle for pipette tips as inaccuracies and loss in precision arise when low surface tension liquids such as viscous oils are pipetted. Here we propose the use of lubricant-infused surface (LIS) technology to achieve omniphobic properties in pipette tips. Using a versatile and simple design, the inner lumen of commercially available pipette tips was coated with a fluorosilane (FS) layer using chemical vapor deposition (CVD). We show that after lubricating the tips through simply pipetting up and down a fluorinated lubricant, the surface free energy of the tips drastically decreased enabling them to attain low retention properties. Contact angle measurements reveals that the treated pipette tips have enhanced omniphobic properties. The repellent behavior of the lubricant-infused pipette tips against physical adsorption is investigated through pipetting a food coloring dye as well as human blood samples and are compared to the untreated tips. The results show significantly less amount carryover residue when the lubricant-infused tips are utilized compared to commercially available ones.

\section{Introduction}

Fluid carryover in liquid handling devices can lead to experiment failure, measurement inaccuracies and sample loss. It is the major cause of cross-contamination in general scientific procedures such as bacteriological work, polymerase chain reaction (PCR), and radioimmunoassay ${ }^{1-4}$. For instance, in PCR amplification reactions in a criminal forensic lab, small amounts of DNA contamination could result in amplifying DNA to promote false positive identifications ${ }^{5}$. In such instances, an underlying cause of contamination could originate from the pipetting of substances with highly viscosities and low surface tensions which can stick to the plastic surface of the pipette, resulting in an improper ejection onto the next test sample ${ }^{6-8}$. Moreover, carryover contamination in pipetting can lead to erroneous volume determination, the need to change tips, limitations to fixed pipettes which require to work with open tubes, carbon footprint and costs associated with disposing of single use tips. In order to substantially minimize the effect of carryover, laboratory work areas need separate sets of supplies and equipment, such as pipettes, test-tube holders, and centrifuges ${ }^{9-11}$. Single-use tips with filters are usually recommended as the main strategy to prevent amplicon contamination originated form amplicons that accumulate within pipettor ${ }^{10,12,13}$. However, single-use tips are not applicable to automated robotic workstations with their use of fixed tips ${ }^{14}$. Techniques have currently been put in place for prevention of cross-contamination, through increasing well-to-well spacing and the prevention of involuntarily z-axes movements from robotic gripper during plate transfer ${ }^{10,15}$. In addition, present literature suggests fixed tips treated with robust washing routines are approving of casework samples and serve as a viable and effective alternative to disposable tips ${ }^{15}$. The issue of carryover contamination can be broadened towards other 
forms of laboratory equipment, such as the syringes and needles used in the removal of PCR products on the robotic arm of automated amplification systems ${ }^{16}$. A potential solution would involve surface modifications to laboratory equipment, namely pipette tips, such that the issue of sample carryover can be minimized.

Most pipette tips on the market are manufactured with hydrophobic polymers that repel high surface tension liquids like water. One of the most commonly used polymers to mold pipette tips is polypropylene due to its hydrophobicity, cost effectiveness, and availability. While hydrophobic and superhydrophobic surfaces are useful for repelling high surface tension liquids, they lack in performance when low surface tension liquids are involved. Thus, this presents an obstacle for pipette tips as inaccuracies and loss in precision arise when low surface tension liquids such as viscous oils are pipetted.

To address this issue, the surface of the tip must be modified such that it exhibits low affinity to low surface tension liquids. Such surfaces, referred to as omniphobic, are characterized by their ability to repel all liquids despite their cohesive force strengths ${ }^{17,18}$. A static contact angle of $90^{\circ}$ or higher accompanied by a low dynamic angle hysteresis and a low sliding angle of $5^{\circ}$ or less are generally characteristic of omniphobic surfaces ${ }^{19,20}$. Omniphobic surfaces can be fabricated using two general techniques: physical and chemical modifications. Physical modification involves roughening the surface using methods such as nano particle deposition, lithographic imprinting and etching ${ }^{21-23}$. Chemical modification lies on the basis of decreasing the free energy of the surface of interest ${ }^{1,24,25}$. Several surface modification techniques that rely on altering the surface chemistry are known, some of the most prevalent include the use of fluorocarbon compounds or organosilanes as surface coatings ${ }^{21,26}$.

The growing demand for pipette tips with minimal sample surface adherence has led to the development of low retention pipette tips. Low retention pipette tips exhibit omniphobic surface properties thereby leading to minimal sample loss. One example involves using unique molding processes to incorporate fluoropolymer molecules into the surface of the pipette tips to achieve hydrophobic properties ${ }^{27}$. Texturing the pipette tips is another technique used by researchers to hydrophobically coat the pipette tips ${ }^{28,29}$. These methods, however, present negative aspects such as increased manufacturing complexity, high cost and lower effectiveness as compared to surface coating.

Here, we propose the use of CVD treatment with a simple apparatus designed to achieve omniophobic properties in pipette tips. CVD treatment of a fluorosilane can drastically reduce the surface free energy of the tip enabling it to attain low retention properties. The fluorosilanized tips are then lubricated with a fluorinated lubricant to generate lubricant infused surfaces (LIS) with omniphobic properties. Some of the benefits of this method include process simplicity, lower processing time, high efficiency and reduced cost.

\section{Materials And Methods}

\subsection{Materials}


Trichloro $(1 \mathrm{H}, 1 \mathrm{H}, 2 \mathrm{H}, 2 \mathrm{H}$-perfluorooctyl) silane (TPFS), perfluoroperhydrophenanthrene (PFPP) (Sigma, Oakville, Canada), pipette tips composed of polypropylene (Diamed Lab Supplies Inc., Mississauga, Canada), red food coloring dye (A Preema Quality Product, Ingredients: Sodium Chloride and E122 Carmosine), and tween 20 (Sigma, Oakville, Canada) were used as received. Citrated human blood was generated from blood samples collected from healthy donors. All procedures were approved by the McMaster University Research Ethics Board. All methods were performed in accordance with the relevant guidelines and regulations. Informed consent was obtained from all the donors.

\subsection{Methods}

Pipette tips were placed onto a cylindrical shaped apparatus acting as a holder device. Holder apparatus was placed in an oxygen plasma cleaner (Harrick Plasma Cleaner, PDC-002, $230 \mathrm{~V}$ ) and exposed to highpressure oxygen plasma for 2 minutes for surface functionalization to enable reaction with TPFS. Upon oxygen plasma-treatment and removal from the plasma cleaner, the holder apparatus with pipette tips and petri dish with control tips samples were placed in a desiccator connected to a vacuum pump. 200 $\mu \mathrm{L}$ of TPFS was pipetted onto a glass slide on a separate petri dish located across from the holder apparatus. $100 \mu \mathrm{L}$ of TPFS was also pipetted on glass slide located inside the holder apparatus (Fig. 1). The desiccator was vacuumed at a pressure of $-0.08 \mathrm{MPa}$, therefore initiating the CVD treatment. The silanization reaction took place over a period of 2.5 hours at room temperature. Upon completion of CVD, pipette tips were removed from the holder device and heat treated at $60^{\circ} \mathrm{C}$ overnight. In order to infuse lubricant in the fluorosilanized pipette tips, the PFPP lubricant was simply pipetted in and out.

FT-IR (Bruker, Karlsruhe, Germany) was used to assess the surface chemical composition of the pipette tips before and after the treatment. During FT-IR measurements, the air was considered the background, and the other spectra of the fluorosilanized and untreated tip surfaces were normalized based on this baseline. Contact angles of the fluorosilanized and untreated were measured using $5 \mu \mathrm{L}$ droplets of water, tween 20, and isopropanol. Water sessile drop contact angle measurements were performed at room temperature before and after each modification step using a Future Digital Scientific OCA20 goniometer (Garden City, NY), which was calibrated prior to each measurement.

The blood cells and proteins interactions with lubricant-infused and untreated pipette tips were assessed with recalcified blood. Citrated human blood was recalcified by $\mathrm{CaCl}_{2}$ diluted in HEPES at a concentration of $12.5 \mathrm{mM}$. For scanning electron microscopic (SEM) imaging (JSM-7000 F), after pipetting up and down the recalcified blood, the tips were cut and fixed using $2 \%$ glutaraldehyde diluted in PBS. The tips were then incubated in $1 \%$ osmium tetroxide in $0.1 \mathrm{~m}$ sodium cacodylate buffer for an hour, followed by dehydration via a graded series of ethanol and critical point drying by Leica EM CPD300 dryer (Leica Mikrosysteme $\mathrm{GmbH}$, Wien, Austria) using liquid $\mathrm{CO}_{2}$ flush. Before imaging, the samples were gold sputtered (Polaron Model E5100 sputter coater, Watford, Hertfordshire).

In the dye experiments, 10X serial dilutions of the red dye were performed in a well-plate to assess carryover of treated tips. The initial concentration of the dye was $1 \mathrm{mg} \mathrm{mL}^{-1}$. The dye was serially diluted in water containing $0.05 \%$ tween 20 using lubricant infused and untreated tips for comparison. For the 
purpose of the carryover evaluation, the pipette tips during the serial dilutions were not changed and the same pipette tip was implemented throughout all the dilution steps. The well-plate was analyzed using a plate reader (Tecan Infinite M1000) to find the absorbance values.

\section{Results And Discussion}

In order to uniformly coat the inner surface of the pipette tips composed of polypropylene (Diamed Lab Supplies Inc.), they were placed onto a cylinder-shaped apparatus which acted as a holding device. The apparatus with tips were oxygen plasma treated and silanized with trichloro $(1 \mathrm{H}, 1 \mathrm{H}, 2 \mathrm{H}, 2 \mathrm{H}-$

perfluorooctyl) silane (TPFS) using the CVD method. This allows the hydrophilic terminal (trichlorosilane) of the fluorosilane molecule to bind to the plasma induced hydroxyls on the surface of the tips, resulting in self-assembled monolayers (SAMs) of fluorosilane (FS) with an umbrella-shaped structure in a way that the fluorine terminals are exposed on the surface (Fig. 1). The fluorinated tips were then lubricated by simply pipetting in and out a fluorinated lubricant called perfluoroperhydrophenanthrene (PFPP).

To analyze the changes in the chemical composition of the pipette tip surface after oxygen plasma treatment and after CVD modification, fourier-transform infrared spectroscopy (FT-IR) was performed on $1 \mathrm{~mL}$ fluorosilanized and untreated pipette tips (Fig. 2a). The spectra of both fluorosilanized and untreated tips had a number of peaks in the absorption band between 800 to $1200 \mathrm{~cm}^{-1}$, specifically at $850 \mathrm{~cm}^{-1}, 1000 \mathrm{~cm}^{-1}$, and $1200 \mathrm{~cm}^{-1}$ vibrations that are characteristic for isotactic polypropylene. These absorption bands can be interpreted as vibrations of $\mathrm{C}-\mathrm{H}, \mathrm{CH}_{2}$ and $\mathrm{CH}_{3}$ groups in the polymer chain ${ }^{30}$. In the fluorosilanized tips, however, a broad absorbance band is present around the $3700-3200 \mathrm{~cm}^{-1}$. This is believed to correspond to the $\mathrm{Si}-\mathrm{OH}$ group bonds formed as a result of the hydrolysis of TPFS molecules. It should be mentioned that $3700-3200 \mathrm{~cm}^{-1}$ is the range of absorbance bands that commonly corresponds to alcohols and phenols, including bonds to silicone compounds ${ }^{30}$. This pattern is notably absent when looking at the untreated tip sample spectra, which produces a flat line over the 4000-3000 $\mathrm{cm}^{-1}$ region (Fig. 2a).

To examine the relative hydrophobicity/hydrophilicity of the treated and untreated pipette tips, contact angle measurements were performed using a $5 \mu \mathrm{L}$ droplet of deionized water. The static contact angle measurements of the untreated tips, fluorosilanized tips, and lubricant infused tips are shown in Fig. 2b. Untreated tips demonstrated a lower contact angle $\left(\theta s t=75 \pm 11^{\circ}\right)$ implying low levels of hydrophobicity. It is worth noting that the standard deviation value of the untreated samples is relatively high due to the conditions of the untreated tip surface. Upon CVD treatment and before lubricant addition, the contact angle increased to $89 \pm 3^{\circ}$. Finally, after addition of PFPP lubricant to the fluorosilanized tips, contact angles of water were demonstrated to increase to $101.7 \pm 7^{\circ}$.

Due to the surface curvature of the pipette tip, measuring the contact angle of a liquid droplet on the tip using a tensiometer presents complications and might result in slight inaccuracy. As such, the contact angle of water, tween 20, and isopropanol as a low surface tension liquid were measured on treated and standard pipette tips using capillary rise method. Capillary rise can be utilized to compute the contact 
angle of the rising/dropping liquid using hydrostatics principles. Due to the close resemblance of the tips to a truncated cone shape, the capillary rise equation was modified to account for such capillary shape (equation 1):

$$
\theta=\cos ^{-1}\left[\frac{\rho g h R}{2 \gamma}\right]-\beta
$$

where $\theta$ is the contact angle, $\rho$ is the density of the liquid, $g$ is the gravitational constant, $h$ is the rise/drop height, $R$ is the diameter of tube at the three-phase region, $\gamma$ is the surface tension of the liquid, and $\beta$ is the conical angle of tip.

As such, the contact angles of liquids with various surface tensions on the fluorosilanized and untreated tips were acquired to test the effect of surface modification on omniphobic properties of the tips (Fig. 2c). The contact angles of all three liquids were higher on the fluorosilanized tips compared to untreated tips. This is especially evident for the lower surface tension liquids such as the tween 20 and isopropanol. The increase in the contact angles of the treated samples highlight the higher surface repellency induced by the treatment. The contact angle of tween 20 was $30^{\circ}$ higher on the fluorosilanized surface as compared to the standard while that of isopropanol was $28^{\circ}$ higher. Since the tips exhibited hydrophobic properties even prior to surface treatment due to the properties of the material used to manufacture the tips, there was a smaller difference in contact angle of water on the treated surface with $6.9^{\circ}$ increase using the capillary rise method and $40^{\circ}$ increase using the optical tensiometer. Notable, the hydrophobic nature of the intact pipette tips was not fully appreciated in Fig. $2 b$ which could be due to the fact that the surface properties of the tips somewhat changed when the tips were cut and flattened for the purpose of the contact angle measurement.

In order to study the efficacy of our surface medication in preventing the physical (non-specific) adsorption of biomolecules and reagents, we first performed a visual test through pipetting in and out a food coloring red dye diluted in water containing $0.05 \%$ tween 20 . As it can be seen in Fig. 3a, the untreated $200 \mu \mathrm{L}$ pipette tip showed a great amount of dye adsorption onto the tip, whereas the lubricant infused tip maintained significantly lower amount of dye mostly at the bottom end of the tip after pipetting out the dye. In the next experiment, we used lubricant infused tips at different sizes of $10 \mu \mathrm{L}$, $200 \mu \mathrm{L}$, and $1 \mathrm{~mL}$ to pipette in and out recalcified citrated human blood. As it is demonstrated in Fig. 3b, the lubricant infused tips in all sizes could effectively suppress blood adhesion and clot formation inside the tip in comparison with the untreated tips. SEM images in Fig. 3c also exhibits the presence of blood cells and clot on the surface of the untreated tips, while the lubricant infused modification significantly reduced the cell attachment and clot formation.

To further quantify the effects of lubricant infused technology on the functionality of the pipette tips, 10fold serial dilutions of $1 \mathrm{mg} \mathrm{mL}^{-1}$ dye were performed in water containing $0.05 \%$ tween 20 . The same pipette tip was used to perform all the dilution steps to better illustrate the effect of dye residue inside the tip during the serial dilutions. Using absorbance measurements (at the wavelength of $512 \mathrm{~nm}$ ), we 
quantified the results obtained from lubricant infused and untreated pipette tips in two sizes of $10 \mu \mathrm{L}$ and $200 \mu \mathrm{L}$ shown in Fig. 4a and b, respectively. The results of the dilutions indicate that the untreated tips result in have significantly higher carryover residue; as a result, the dilutions did not occur efficiently. Thus, the more amount of dye that can be seen at lower concentrations could be due to the dye residue that had remained onto the tip surface from the initial high concentrations and was carried to the low concentrations.

As can be seen from the Fig. $4 a$ and $b$, the lubricant infused tips show a steeper decrease in absorbance per dilution number followed by a sooner levelling off to the diluent's absorbance value (with zero concentration of the dye) that is indicated by dashed line in Fig. 4a. On the other hand, the untreated tips reveal higher absorbance levels even for higher dilution numbers which is a result of the sample adhering to the tip's wall even after performing several dilutions. In addition, there is significantly higher variation observed with untreated tips compared to treated ones as indicated by large error bars shown in Fig. 4a and b. In Fig. 4c, the colour difference in the well-plate after performing the serial dilutions via the lubricant infused tips and untreated tips could be observed.

\section{Conclusions}

Sample adherence to tip wall during pipetting activities introduces many problems downsizing experimental accuracy. Current techniques to reduce this effect are expensive and require modifications to the plastic mix used for manufacturing the pipette tips. This work proposes a simple surface modification technique to induce liquid/protein repellency on the inner and outer walls of the pipette tips. The proposed lubricant infused tips with omniphobic properties are able to repel low and high surface tension liquids. The coating will reduce sample carryover and adherence to the tip wall. This technique can thus be used to minimize cross-contamination of experiments involving highly vicious surfaces such as clotting blood and increase efficiency by decreasing sample loss due to carry over adherence.

\section{Declarations}

\section{Acknowledgements}

This work was supported by the NSERC Discovery Grant, Ontario Early Researcher Award and McMaster Start-up funds to TFD. T.F.D is Tier II Canada Research Chair. The electron microscopy was carried out at the Canadian Center for Electron Microscopy (CCEM), a national facility supported by the NSERC and McMaster.

\section{Author Contributions}

H.Y and A.S equally contributed to this work. T.D supervised the research. H.Y and A.S performed the experiments, data validation, data visualization, and analysis, and wrote the manuscript with help from 
S.K and D.A.M, and inputs from M.R. and T.D. All authors have read and approved the final version of the paper.

\section{Competing interest}

The authors declare that they have no known competing financial interests or personal relationships that could have appeared to influence the work reported in this paper.

\section{Data availability}

The datasets generated and analysed during the current study are available from the corresponding author on reasonable request.

\section{References}

1. Le Rouzic, E. Contamination-pipetting: relative efficiency of filter tips compared to Microman® positive displacement pipette. Nat. Methods, 3, III-IV (2006).

2. Schmidt, T., Hummel, S. \& Herrmann, B. Evidence of contamination in PCR laboratory disposables., 82, 423-431 (1995).

3. Buehring, G. C., Eby, E. A. \& Eby, M. J. Cell line cross-contamination: how aware are Mammalian cell culturists of the problem and how to monitor it? In Vitro Cell. Dev. Biol. Anim, 40, 211-215 (2004).

4. Rongxing, W. \& Junhua, Y. D. N. A. Contamination and Treatment Countermeasures in Dog DNA Testing Laboratory DNA Testing. Anim. Husb. Feed Sci, 9, 352-354 (2017).

5. Stinson, L. F., Keelan, J. A. \& Payne, M. S. Identification and removal of contaminating microbial DNA from PCR reagents: impact on low-biomass microbiome analyses. Lett. Appl. Microbiol, 68, 2-8 (2019).

6. Chen, B. K. \& Sun, Y. A MEMS microgripper with changeable gripping tips. in 2011 16th International Solid-State Sensors, Actuators and Microsystems Conference 498-501 (2011)

7. Hartley, J. L. \& Rashtchian, A. Dealing with contamination: enzymatic control of carryover contamination in PCR. PCR Methods Appl, 3, S10-4 (1993).

8. Pierson, S. A. et al. Synthesis and characterization of low viscosity hexafluoroacetylacetonate-based hydrophobic magnetic ionic liquids. New J. Chem, 41, 5498-5505 (2017).

9. Millar, B. C., Xu, J. \& Moore, J. E. Risk assessment models and contamination management: Implications for broad-range ribosomal DNA PCR as a diagnostic tool in medical bacteriology. $J$. Clin. Microbiol, 40, 1575-1580 (2002).

10. Borst, A., Box, A. T. A. \& Fluit, A. C. False-Positive Results and Contamination in Nucleic Acid Amplification Assays: Suggestions for a Prevent and Destroy Strategy. Eur. J. Clin. Microbiol. Infect. Dis, 23, 289-299 (2004).

11. Soltani, E., Rezaee, M. A. \& Gholizadeh, P. How to Reuse Pipette Tips and Tubes in PCR and Electrophoresis Procedures? A New In-house Method Development. J. Res. Med. Dent. Sci, 7, 210- 
213 (2019).

12. Watson, J. et al. Extraction, Identification, and Functional Characterization of a Bioactive Substance From Automated Compound-Handling Plastic Tips. J. Biomol. Screen, 14, 566-572 (2009).

13. Hu, Y. Regulatory concern of polymerase chain reaction (PCR) carryover contamination. in Polymerase Chain Reaction for Biomedical Applications (ed. Samadikuchaksaraei, A.) 57-69 (BoD Books on Demand, 2016)

14. Alexovič, M., Dotsikas, Y., Bober, P. \& Sabo, J. Achievements in robotic automation of solvent extraction and related approaches for bioanalysis of pharmaceuticals. J. Chromatogr. B, 1092, 402421 (2018).

15. Mifflin, T. E., Estey, C. A. \& Felder, R. A. Robotic automation performs a nested RT-PCR analysis for HCV without introducing sample contamination. Clin. Chim. Acta, 290, 199-211 (2000).

16. Lorenz, M. G. O. Liquid-Handling Robotic Workstations for Functional Genomics. JALA J. Assoc. Lab. Autom, 9, 262-267 (2004).

17. Shakeri, A. et al. Antibody Micropatterned Lubricant-Infused Biosensors Enable Sub-Picogram Immunofluorescence Detection of Interleukin 6 in Human Whole Plasma. Small, 16, 2003844 (2020).

18. Badv, M. et al. Biofunctional Lubricant-Infused Vascular Grafts Functionalized with Silanized Bio-Inks Suppress Thrombin Generation and Promote Endothelialization. ACS Biomater. Sci. Eng, 5, 64856496 (2019).

19. Frégeau, C. J., Lett, M., Elliott, C., Yensen, J., Fourney, R. M. \& C. \& Automated Processing of Forensic Casework Samples Using Robotic Workstations Equipped with Nondisposable Tips: Contamination Prevention. J. Forensic Sci, 53, 632-651 (2008).

20. Osborne, M., Aryasomayajula, A., Shakeri, A., Selvaganapathy, P. R. \& Didar, T. F. Suppression of Biofouling on a Permeable Membrane for Dissolved Oxygen Sensing Using a Lubricant-Infused Coating. ACS Sensors, 4, 687-693 (2019).

21. Aslanzadeh, J. Preventing PCR amplification carryover contamination in a clinical laboratory. Ann. Clin. Lab. Sci, 34, 389-396 (2004).

22. Shakeri, A. et al. Self-Cleaning Ceramic Tiles Produced via Stable Coating of TiO2 Nanoparticles. Materials (Basel), 11, 1003 (2018).

23. Imani, S. M. et al. Hierarchical Structures, with Submillimeter Patterns, Micrometer Wrinkles, and Nanoscale Decorations, Suppress Biofouling and Enable Rapid Droplet Digitization. Small, 16, 2004886 (2020).

24. Shakeri, A., Jarad, N. A., Leung, A., Soleymani, L. \& Didar, T. F. Biofunctionalization of Glass- and Paper-Based Microfluidic Devices: A Review. Adv. Mater. Interfaces, 6, 1900940 (2019).

25. Shakeri, A. et al. 26 - Biofunctional interfaces for cell culture in microfluidic devices. in Bioelectronics and Medical Devices: From Materials to Devices - Fabrication, Applications and Reliability (eds. Pal, K.)635-699 (Woodhead Publishing, 2019). doi:https://doi.org/10.1016/B978-0-08-102420-1.00033-9 
26. Shakeri, A., Khan, S. \& Didar, T. F. Conventional and emerging strategies for the fabrication and functionalization of PDMS-based microfluidic devices. Lab Chip, 21, 3053-3075 (2021).

27. Root, D. \& Lawton, L. Sterile irradiated hydrophobic pipette tip. (1993)

28. Kirste, V., Nay, R. \& Beckbissinger, R. Process for hydrophobically coating a pipette tip. (2010)

29. Kirste, V., Nay, R. \& Beckbissinger, R. Pipette tip having a hydrophobic surface texture. (2010)

30. Socrates, G. Infrared and Raman Characteristic Group Frequencies: Tables and Charts (John Wiley \& Sons, 2004).

\section{Figures}




\section{$\mathrm{O}_{2}$ plasma treatment of tips using a holder}

$\_$Pipette tips

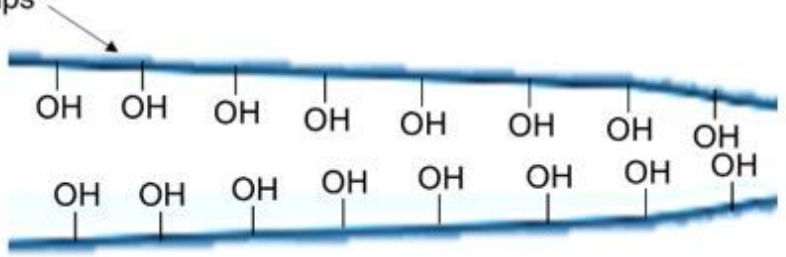

\section{CVD fluorosilanization and heat treatment of tips}
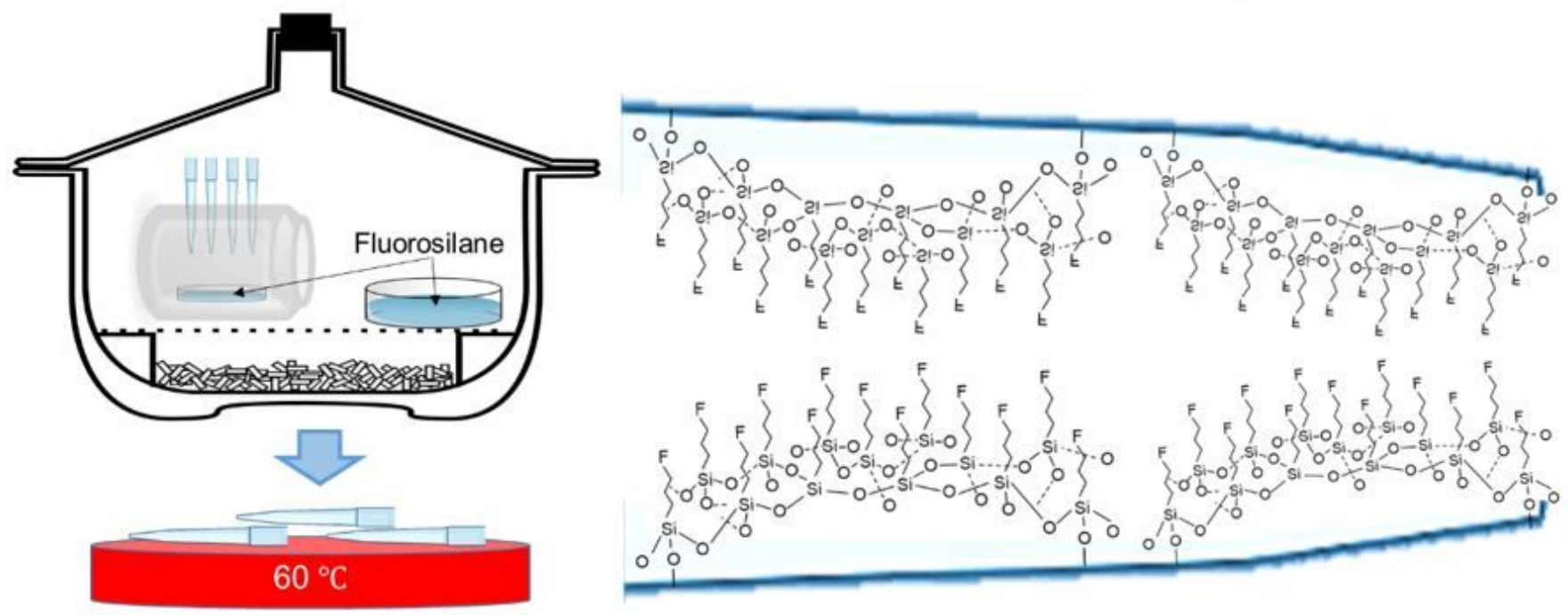

\section{Lubrication of tips}
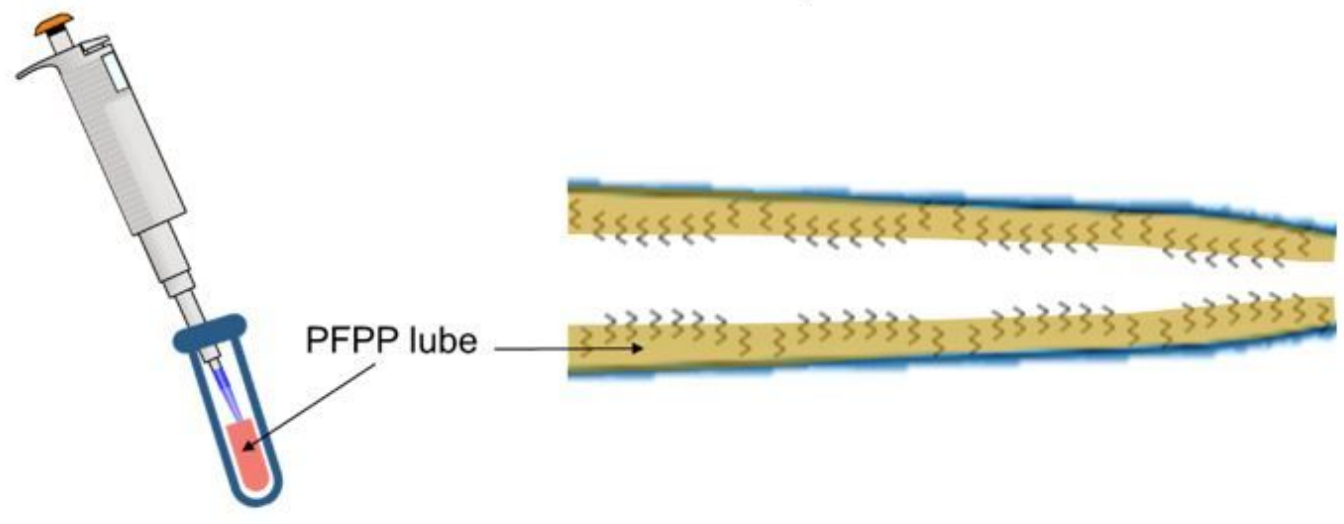

\section{Figure 1}

Schematic illustration of the treatment procedure along with the chemical structures of the treated tips at each step of the modification. Pipette tips in specially designed holder apparatus were CVD treated with TPFS followed by heat treatment at $60^{\circ} \mathrm{C}$ overnight. The tips were subsequently lubricated via pipetting in and out PFPP lube. 
(a)

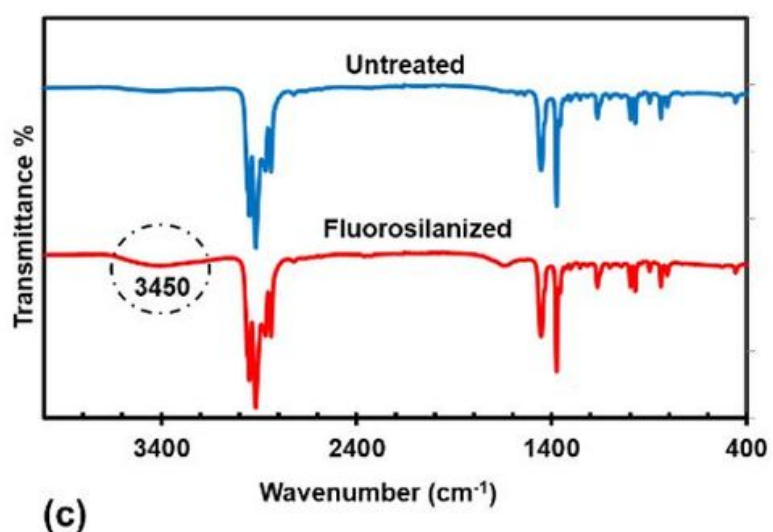

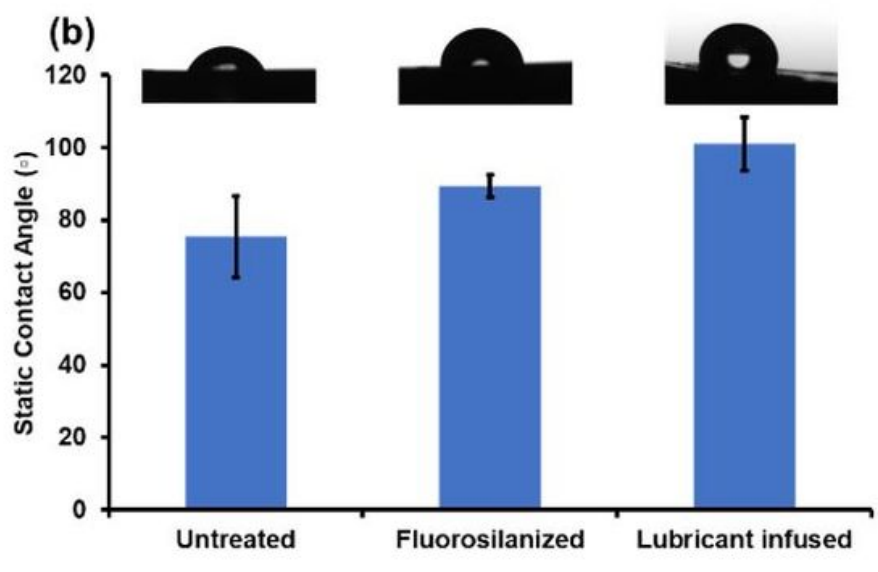

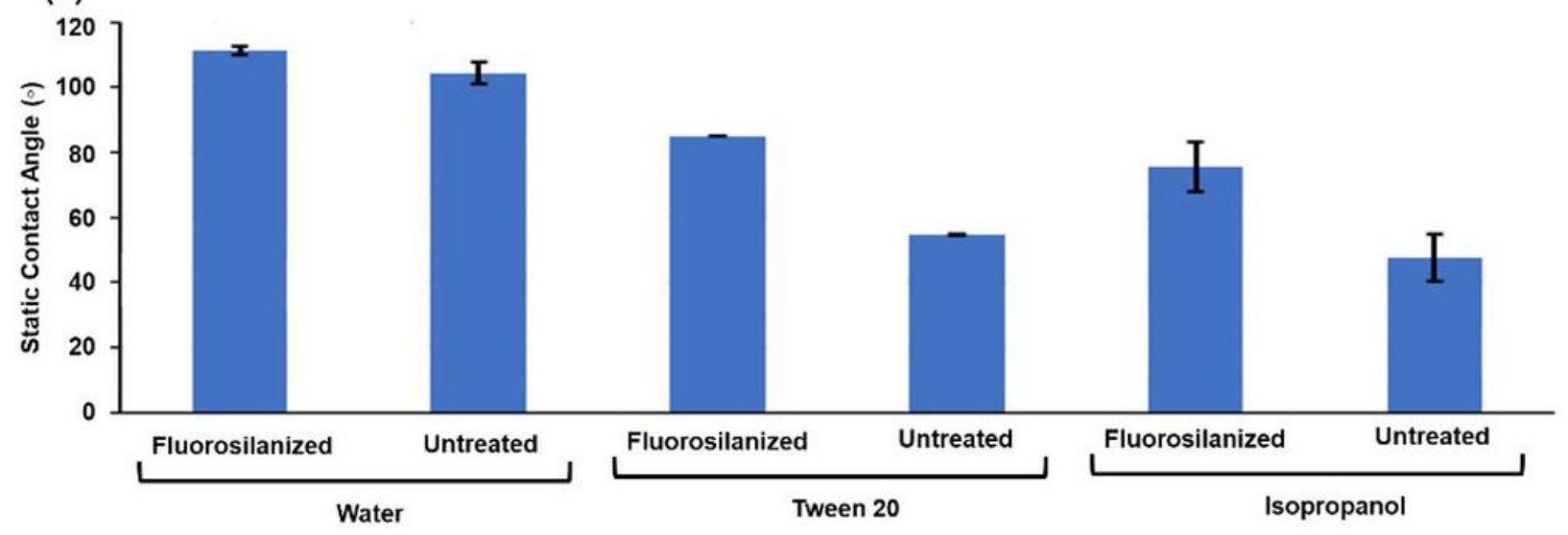

Figure 2

(a) FTIR spectra of an untreated pipette tip compared to a fluorosilanized tip. Circled broad peak in 3700$3200 \mathrm{~cm}-1$ region indicates presence of $\mathrm{Si}-\mathrm{OH}$ bonds. (b) Static contact angle measurement of untreated, fluorosilanized, and lubricated pipette tip samples. (c) Static contact angle comparison between water, tween 20 , and isopropanol measured using capillary pressure. All results are presented as means \pm S.D. 
(a)

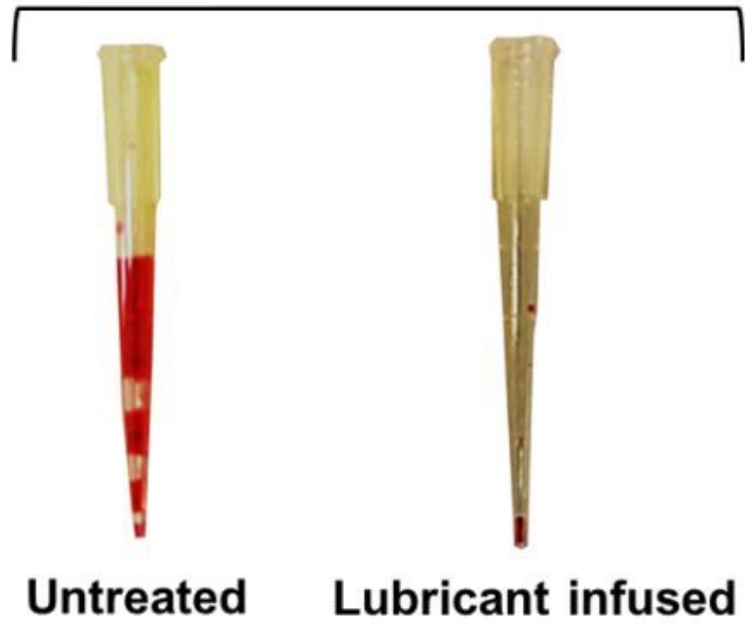

(c)

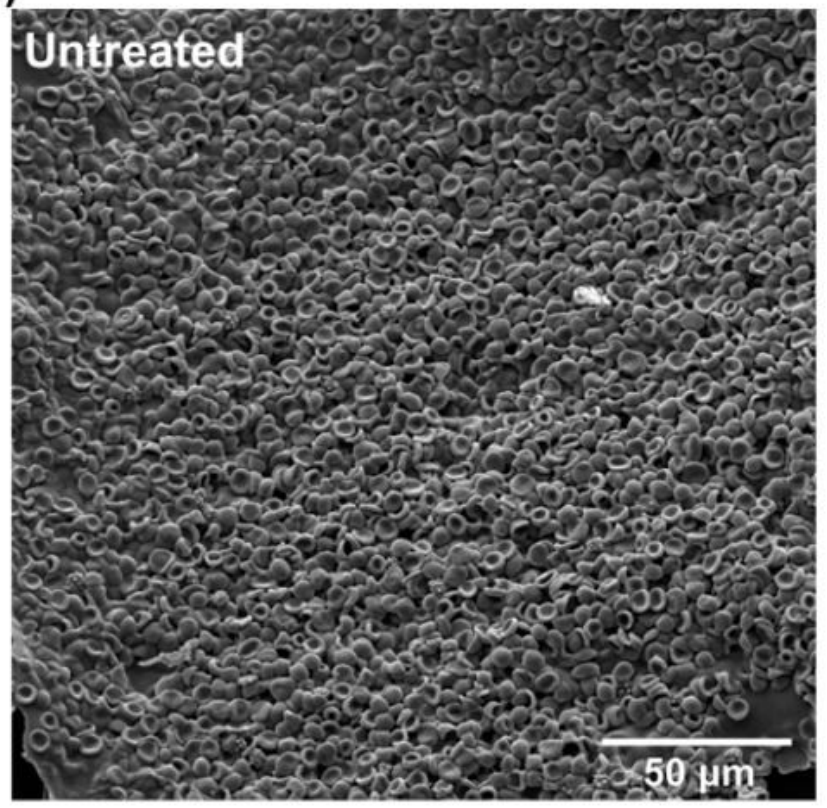

(b)

\section{Blood}

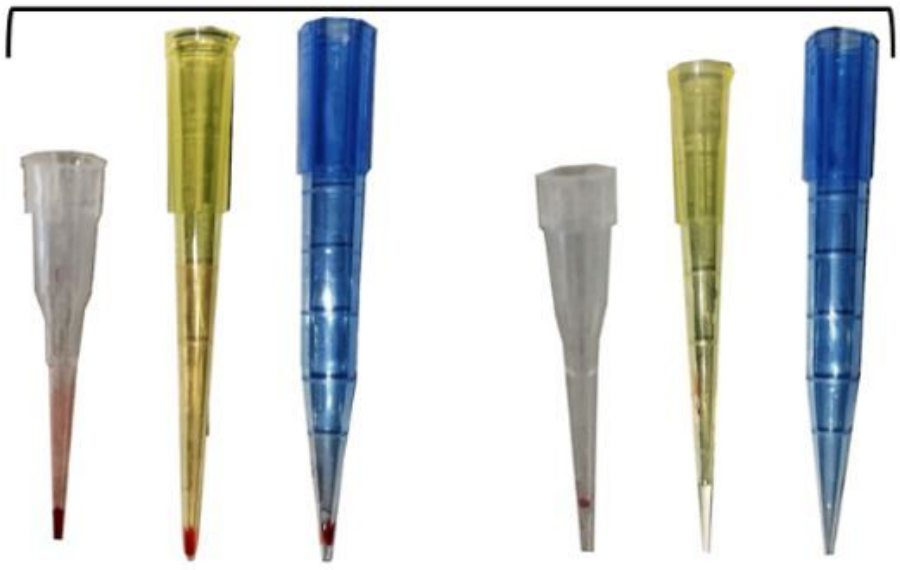

Untreated

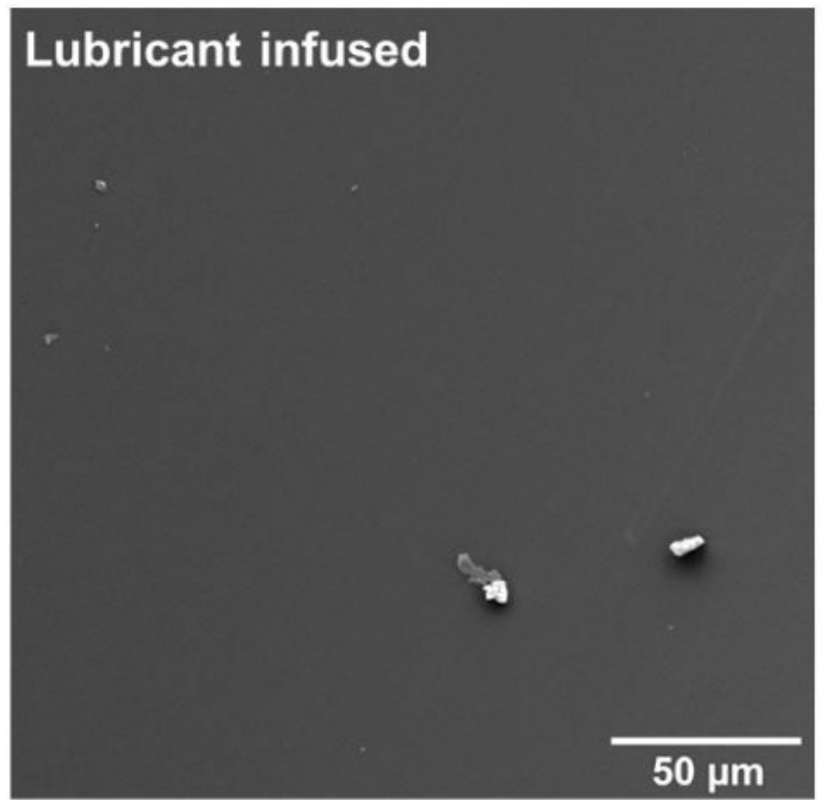

Figure 3

(a) Comparison of the red dye attachment to $200 \mu \mathrm{L}$ untreated tips and fluorosilanized lubricant infused tips. (b) Comparison of the clotting blood attachment to $10 \mu \mathrm{L}, 200 \mu \mathrm{L}$, and $1000 \mu \mathrm{L}$ untreated tips and fluorosilanized lubricant infused tips. (c) SEM images of clot attached to the surface of the untreated tip compared to the fluorosilanized lubricant infused tip. 
(a)

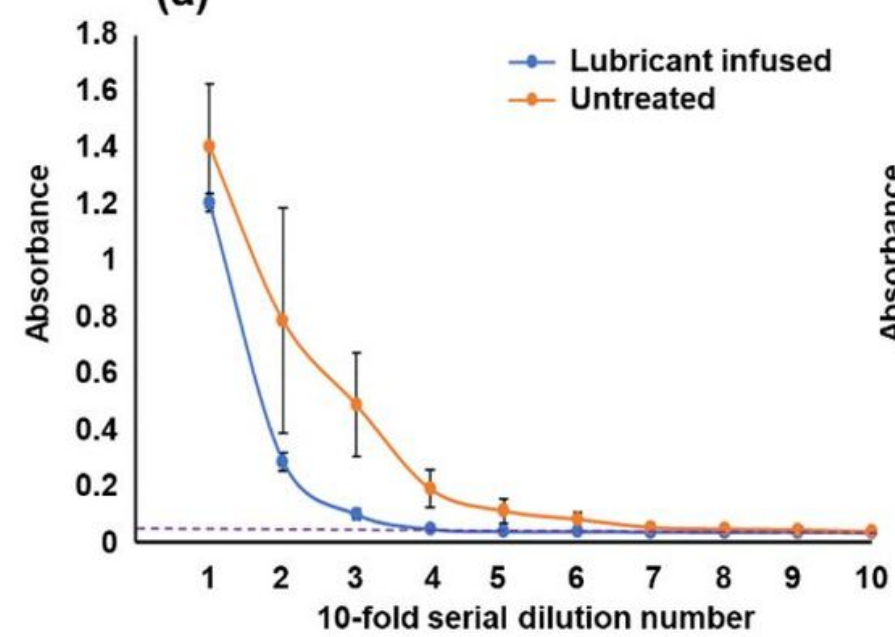

(b)

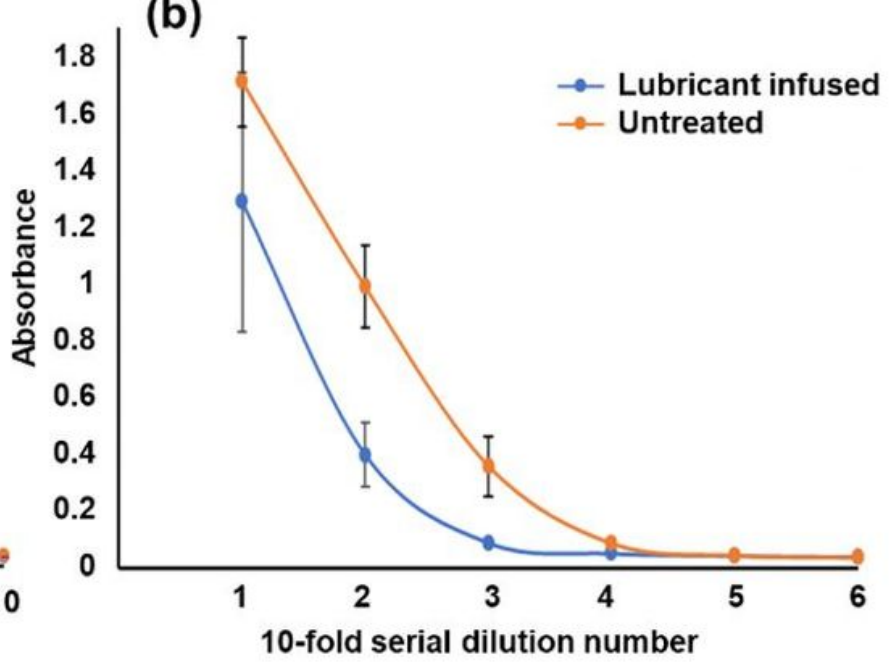

(c)

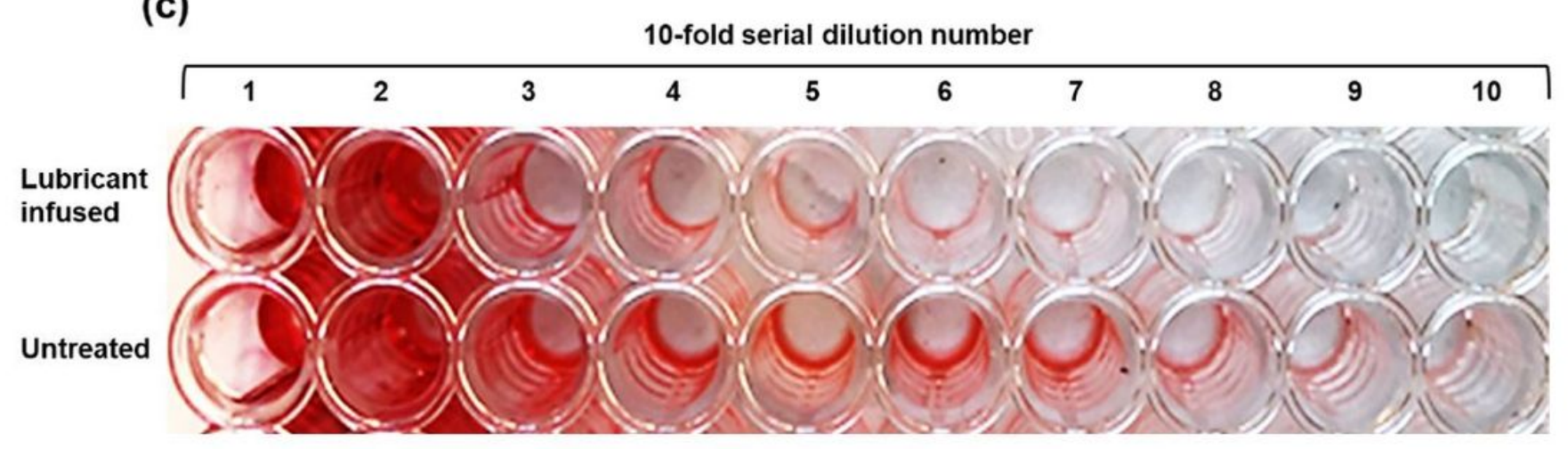

Figure 4

Absorbance of the red dye at different concentration after performing 10-fold serial dilutions using (a) 10 $\mu \mathrm{L}$ and (b) $200 \mu \mathrm{L}$ untreated tips compared to fluorosilanized lubricant infused tips. For each experiment a single tip was used for all the serial dilutions. Dashed line in (a) represents the absorbance at zero concentration of the red dye. The results are presented as means \pm S.D. (c) Serial dilutions of the red dye in a well plate using single $10 \mu \mathrm{L}$ untreated and lubricant infused tips. Untreated wells showed higher residue carryover of dye, as dilution did not occur effectively. 